\title{
Age at menarche and age at natural menopause in East Asian women: a genome-wide association study
}

\author{
Jiajun Shi • Ben Zhang • Ji-Yeob Choi • Yu-Tang Gao • Huaixing Li • Wei Lu • \\ Jirong Long • Daehee Kang • Yong-Bing Xiang • Wanqing Wen • Sue K. Park • \\ Xingwang Ye • Dong-Young Noh • Ying Zheng • Yiqin Wang • Seokang Chung • Xu Lin • \\ Qiuyin Cai • Xiao-Ou Shu
}

Received: 25 November 2015 /Accepted: 14 July 2016/Published online: 14 September 2016

(C) American Aging Association 2016

\begin{abstract}
Age at menarche (AM) and age at natural menopause (ANM) are complex traits with a high heritability. Abnormal timing of menarche or menopause is associated with a reduced span of fertility and risk for several age-related diseases including breast, endometrial and ovarian cancer, cardiovascular disease, and osteoporosis. To identify novel genetic loci for AM or ANM in East Asian women and to replicate previously identified loci primarily in women of European ancestry by
\end{abstract}

Electronic supplementary material The online version of this article (doi:10.1007/s11357-016-9939-5) contains supplementary material, which is available to authorized users.

J. Shi · B. Zhang $\cdot$ J. Long $\cdot$ W. Wen · Q. Cai ·

X.-O. Shu $(\bowtie)$

Department of Medicine, Vanderbilt Epidemiology Center and Division of Epidemiology, Vanderbilt University School of Medicine, 2525 West End Avenue, Suite 600, IMPH, Nashville, Tennessee 37203, USA

e-mail: xiao-ou.shu@vanderbilt.edu

J.-Y. Choi $\cdot$ D. Kang $\cdot$ S. K. Park $\cdot$ S. Chung

Department of Biomedical Sciences, Seoul National University

College of Medicine, Seoul, Korea

J.-Y. Choi · D. Kang · S. K. Park

Department of Preventive Medicine, Seoul National University

College of Medicine, Seoul, Korea

J.-Y. Choi $\cdot$ D. Kang $\cdot$ S. K. Park

Cancer Research Institute, Seoul National University, Seoul, Korea genome-wide association studies (GWASs), we conducted a two-stage GWAS. Stage I aimed to discover promising novel AM and ANM loci using GWAS data of 8073 women from Shanghai, China. The Stage II replication study used the data from another Chinese GWAS $(n=$ 1230 for AM and $n=1458$ for ANM), a Korean GWAS ( $n=4215$ for AM and $n=1739$ for ANM), and de novo genotyping of 2877 additional Chinese women. Previous GWAS-identified loci for AM and ANM were also

Y.-T. Gao • Y.-B. Xiang

Department of Epidemiology, Shanghai Cancer Institute, Renji

Hospital, Shanghai Jiaotong University School of Medicine,

Shanghai, China

H. Li $\cdot$ X. Ye $\cdot$ Y. Wang $\cdot$ X. Lin

Key Laboratory of Nutrition and Metabolism, Institute for Nutritional Sciences, Shanghai Institutes for Biological Sciences, Chinese Academy of Sciences and Graduate School of the Chinese Academy of Sciences, Shanghai, China

W. Lu • Y. Zheng

Shanghai Municipal Center for Disease Control and Prevention, Shanghai, China

D.-Y. Noh

Department of Surgery, Seoul National University College of Medicine, Seoul, Korea 
evaluated. We identi- fied two suggestive menarcheal age loci tagged by rs79195475 at 10q21.3 (beta $=$ -0.118 years, $P=3.4 \times 10^{-6}$ ) and $\mathrm{rs} 1023935$ at $4 \mathrm{p} 15.1$ (beta $=-0.145$ years, $P=4.9 \times 10^{-6}$ ) and one menopausal age locus tagged by rs3818134 at 22q12.2 (beta $=$ -0.276 years, $P=8.8 \times 10^{-6}$ ). These suggestive loci warrant a further validation in independent populations. Although limited by low statistical power, we replicated 19 of the 98 menarche loci and 5 of the 20 menopause loci previously identified in women of European ancestry in East Asian women, suggesting a shared genetic architecture for these two traits across populations.

Keywords Menarche Menopause - Genome-wide association $\cdot$ Single nucleotide polymorphism

\section{Introduction}

Age at menarche (AM) and age at natural menopause (ANM) indicate the beginning and end of a woman's normal reproductive life. Menarche and menopause out of the common range have been associated with risk for various diseases, including breast cancer (Velie et al. 2005; Vogel 2008), endometrial and ovarian cancer (Hinds and Price 2010; Cramer 2012), cardiovascular disease (Cui et al. 2006), and osteoporosis (Qiu et al. 2013; Parker et al. 2014). It has been well recognized that genetic makeup influences both AM (Sharma 2002; Anderson et al. 2008) and ANM (de Bruin et al. 2001; Murabito et al. 2005; Morris et al. 2011). Recent genome-wide association studies (GWASs) among women of European ancestry have identified at least 106 genetic loci for AM (He et al. 2009; Ong et al. 2009; Perry et al. 2009; Sulem et al. 2009; Elks et al. 2010; Perry et al. 2014) and 21 loci for ANM (He et al. 2009; Stolk et al. 2009; Stolk et al. 2012). However, only 24 AM loci and 9 ANM loci have been replicated in women of either Asian or African ancestry and no novel loci have been identified in these non-European populations (Liu et al. 2009; Chen et al. 2012; Shen et al. 2013; Spencer et al. 2013; Tanikawa et al. 2013; Delahanty et al. 2013; Carty et al. 2013; Demerath et al. 2013; Pyun et al. 2014; Chen et al. 2014).

We report here results of a meta-analysis of GWAS for AM and ANM in Chinese and Korean populations, aiming to replicate the AM and ANM loci identified in European populations and to discover novel genetic loci.

\section{Methods}

\section{Study design}

This GWAS included two stages. The Stage I study was a meta-analysis of results of AM and ANM association using data generated from the Shanghai Genome-Wide Association Studies (SGWAS), which included female participants of the Shanghai Breast Cancer Genetics Study (SBCS) (Zheng et al. 2009), the Shanghai Endometrial Cancer Study (SECS) (Long et al. 2012), the Shanghai Type II Diabetes Studies (ST2DS) (Shu et al. 2010), the Shanghai Colorectal Cancer Study (SCRCS) (Jia et al. 2013; Zhang et al. 2014), and other ancillary studies using the Shanghai Women's Health Study (SWHS) samples (Petersen et al. 2010; Abnet et al. 2010). All these studies, except for the SBCS and SECS, drew women from the SWHS, a population-based cohort study (Zheng et al. 2005). We defined a suggestive locus as a genomic region wherein one or more singlenucleotide polymorphisms (SNPs) showed $P<1 \times 10^{-4}$ in Stage I and the region was $>1 \mathrm{Mb}$ away from GWAS locus discovered in women of European ancestry. One or two highly correlated SNPs $\left(r^{2}>0.6\right)$ in each suggestive novel locus were selected for a replication study using data from three additional studies in Chinese and Korean women (see description in Stage II). Index SNPs or their best proxies of AM or ANM loci identified by previous GWAS in European ancestry women were also evaluated in the Stage II study. Basic information on all participating studies is summarized in Table 1.

Stage I samples and genotyping

A total of 8073 Chinese women from the SGWAS were included in Stage I (Table 1). These studies were population-based and applied similar study protocols to collect blood or buccal cell samples and relevant exposure information. Interviews were conducted in person by trained, retired medical personnel, and anthropometrics, including height, weight, and circumferences of the waist and hips were measured by trained interviewers according to standard protocols. AM, to the nearest year, was ascertained by participant recall during the interview. We used the World Health Organization's definition of menopause, i.e., the cessation of menstruation for $\geq 12$ months, to determine menopausal status. Information on age at which menopause occurred and the reasons for its occurrence (natural menopause, 
Table 1 Characteristics of participants in the association of age at menarche and natural menopause

\begin{tabular}{|c|c|c|c|c|c|c|}
\hline \multirow[t]{2}{*}{ Study (acronym) } & \multirow[t]{2}{*}{ Population } & \multirow[t]{2}{*}{ Primary trait } & \multicolumn{2}{|c|}{ Age at menarche } & \multicolumn{2}{|c|}{$\begin{array}{l}\text { Age at natural } \\
\text { menopause }\end{array}$} \\
\hline & & & $n$ & Mean (SD) & $n$ & Mean (SD) \\
\hline \multicolumn{7}{|l|}{ Stage I: Initial Shanghai GWAS meta-analysis } \\
\hline \multirow[t]{2}{*}{ The Shanghai Breast Cancer Study (SBCS) } & Chinese & Breast cancer cases & 2697 & $14.5(1.7)$ & 821 & $50.0(3.6)$ \\
\hline & & Healthy controls ${ }^{\mathrm{a}}$ & 1987 & $14.7(1.8)$ & 684 & $49.1(4.0)$ \\
\hline The Shanghai Endometrial Cancer Study (SECS) & Chinese & Endometrial cancer cases & 827 & $14.5(1.7)$ & 457 & $50.4(3.4)$ \\
\hline The Shanghai Type II diabetes Studies (ST2DS) & Chinese & T2D cases & 1030 & $14.7(1.8)$ & 496 & $49.3(3.5)$ \\
\hline \multirow[t]{2}{*}{ The Shanghai Colorectal Cancer Study (SCRCS) } & Chinese & CRC cases & 487 & $15.1(1.8)$ & 328 & $49.2(3.6)$ \\
\hline & & Healthy controls & 730 & $15.2(1.8)$ & 531 & $49.5(3.4)$ \\
\hline Other Shanghai cancer studies & Chinese & Other cancer cases ${ }^{\mathrm{b}}$ & 315 & $15.3(1.7)$ & 239 & $48.9(3.7)$ \\
\hline Stage I total & & & 8073 & & 3556 & \\
\hline \multicolumn{7}{|l|}{ Stage II: replication studies } \\
\hline $\begin{array}{l}\text { Nutrition and Health of Aging Population in } \\
\text { China (NHAPC) }\end{array}$ & Chinese & Aging & 1230 & $15.9(2.1)$ & 1458 & $48.9(4.0)$ \\
\hline \multirow[t]{2}{*}{ The Seoul Breast Cancer Study (SeBCS) } & Korean & Breast cancer cases & 2164 & $14.7(1.7)$ & 745 & $48.4(5.5)$ \\
\hline & & Healthy controls & 2051 & $15.2(1.8)$ & 994 & $49.2(4.6)$ \\
\hline Shanghai Women's Health Study (SWHS) & Chinese & Healthy women $^{\mathrm{c}}$ & 2877 & $15.1(1.8)$ & 1911 & $49.0(4.1)$ \\
\hline Stage II total & & & 8322 & & 5108 & \\
\hline Stage I and II total & & & 16,395 & & 8664 & \\
\hline
\end{tabular}

${ }^{a}$ Note shared controls in GWAS in Stage I; most samples were selected from the Shanghai Women's Health Study (Zheng et al., 2005)

${ }^{\mathrm{b}}$ These include 219 cases from the Shanghai Upper Gastrointestinal Cancer Study (SUGICS), 46 cases from the Shanghai Pancreatic Cancer Study (SPCS), and 50 cases with other cancers

${ }^{\mathrm{c}}$ Not included in Stage I GWAS, with genotype data available for several genetic variants noted in Table 2

hysterectomy or ovariectomy, or other treatmentinduced menopause) were obtained in survey interviews. ANM was determined by subtracting the birth date from the date of the last natural menstrual period. Women with a non-natural cause of menopause (i.e., menopause brought on by surgery, radiation treatment, or chemotherapy) were excluded. The details on study design and participant characteristics for the SGWAS and the SWHS have been described elsewhere (Zheng et al. 2005; Wen et al. 2014). Written informed consent was obtained from all participants prior to interview, and the study protocols were approved by the institutional review boards of all institutions involved in the study.

Genotyping methods and quality control (QC) in the SGWAS were previously described in detail (Zheng et al. 2009; Wen et al. 2014). In brief, genotyping was performed using either Affymetrix or Illumina SNP arrays. For the present study, QC procedures included the removal of SNPs with minor allele frequency (MAF) of $<5 \%$, Hardy-Weinberg equilibrium (HWE) $P$ values of $<1 \times$
$10^{-5}$ and samples with $>5 \%$ missing genotypes, outliers from multidimensional scaling analyses based on pairwise identity-by-state (IBS), or duplicates and first-degree relatives based on identity-by-descent (IBD) analysis. After QC filtering, genotype imputation was performed by each participating study using MACH 1.0 (Li et al. 2010) with the Genetic Investigation of ANthropometric Traits (GIANT) all-reference panel (excluding monomorphic and singleton sites) from the 1000 Genomes Project phase 1, release v3. A total of 4,633,105 SNPs with an imputation score of Rsq $>0.5$ and with MAF $>5 \%$ were included in final AM or ANM association analysis.

Stage I statistical analysis

A linear regression model, adjusted for age, disease status (e.g., cancer or type II diabetes), and the first ten principal components from population structure analysis using linkage disequilibrium (LD)-pruned (pairwise $r^{2}$ $<0.2$ ) common SNPs with MAF $>0.3$, was used to 
evaluate association between SNPs and AM or ANM in each subset of the GWAS using mach2qtl software (Li et al. 2009; Li et al. 2010). Fixed-effect inverse-variance weighting meta-analysis was performed with summary statistics of beta (per-allele effect on AM or ANM) and standard error from all GWAS using the METAL software (Willer et al. 2010). The presence of heterogeneity across studies was tested with Cochran's $Q$ statistics implemented in METAL. The inflation factor was modest $(\lambda=1.009)$ using all SNPs, suggesting little evidence of population stratification in our studies.

Stage II samples, genotyping, and association with AM or ANM

The Seoul Breast Cancer Study (SeBCS) is a hospitalbased case-control study conducted in two teaching hospitals in Seoul (Cho et al. 2009; Kim et al. 2012). Included in this project were 2164 incident breast cancer patients recruited between 2001 and 2007. In-person interviews were conducted to collect information on known breast cancer risk factors, including AM and ANM, and anthropometrics by using a protocol and questionnaire. Controls were 2051 women selected from a large urban cohort that is participating in the Korea Genome Epidemiology Study (KoGES), which is an ongoing cohort study seeking to understand the causes and risk factors of disease in Korea. These controls were frequency-matched to cases on age in 5-year intervals. Information on AM and ANM, demographics, and other lifestyle factors were collected using a protocol similar to the SeBCS. DNA from these samples was genotyped using Affymetrix human SNP array 6.0. A QC procedure similar to the one used in the Stage I GWAS was conducted in this study, except for the removal of SNPs with a HWE $P<1 \times 10^{-4}$. Finally, SNPs with genotype imputation Rsq $>0.5$ were analyzed for AM or ANM association using a linear regression model with age, disease status, and the first five population structure informative principal components as covariates.

The Nutrition and Health of Aging Population in China (NHAPC) is a population-based study, focused on investigating the association of environmental and genetic factors with metabolic diseases. A total of 1638 women aged 50-70 years participated in the original study. Details of the study design and inclusion/exclusion criteria have been described elsewhere (Ye et al. 2007). Data on demographic variables including AM $(n=1230)$ and ANM $(n=$ 1458 ) were collected using a standardized questionnaire.
Genotyping was performed using Illumina Human660W arrays. SNPs with MAF $<1 \%$ and subjects with a genotype call rate of $<97 \%$ were removed. Genotype imputation was performed using IMPUTE v2.3.0 with all-phased 1000 Genome haplotypes version 3 as reference (Howie et al. 2009). AM or ANM association was conducted using SNPTEST v2.4.1 (Marchini et al. 2007). The association models included age, region of residence (Beijing or Shanghai), and the first five population structure informative principal components as covariates.

Two suggestive AM- and one ANM-associated SNPs from Stage I were further examined by genotyping using the Sequenom MassARRAY iPLEX platform (Agena Bioscience, San Diego, CA, USA) in an additional 2877 healthy women from the SWHS. SNP association with $\mathrm{AM}$ or ANM was measured under a linear regression model adjusted for age using SAS software, version 9.3 (SAS Institute Inc., Cary, NC, USA).

Stage II meta-analysis

Summary results of AM or ANM association were obtained for each study included in Stage II and conformed to the association direction according to effective alleles revealed in Stage I and then meta-analyzed using METAL (Willer et al. 2010). Results from all samples were obtained through METAL analysis, combining summary results from Stages I and II. The presence of heterogeneity between cohorts for the effect sizes of risk alleles was investigated using Cochran's $Q$ test statistic as implemented in METAL (Willer et al. 2010).

\section{Binomial sign test}

To evaluate consistency of the direction of association for the AM- or ANM-associated GWAS SNPs previously identified in women of European ancestry in our study of women with East Asian ancestry, we performed a binomial sign test. Under the null hypothesis that none of these SNPs/loci are associated with AM or ANM in East Asian populations, half of these evaluated SNPs/ loci would be expected to have an association in the same direction as that of European-ancestry populations. We also conducted a binomial sign test to evaluate the probability of the number of observed significant results. Under the null hypothesis, five percent of evaluated GWAS SNPs would be expected by chance to be associated with the study phenotype at $P<0.05$ and in the same direction as those previously reported. 


\section{Results}

\section{GWAS of AM and ANM}

We identified 37 SNPs at 23 suggestive loci associated with AM and 32 SNPs at 26 suggestive loci associated with ANM at $P<1 \times 10^{-4}$ from the Stage I study (Supplementary Tables $\mathrm{S} 1$ and $\mathrm{S} 2$ ). The association direction of these SNPs was consistent across the GWAS sample sets (Tables S1 and S2). In Stage II, however, none of these suggestive SNPs, except for rs79195475, were significantly associated with AM or ANM in either the healthy Chinese women or Korean women set at $P<0.05$ (Tables $\mathrm{S} 1$ and $\mathrm{S} 2$ ). In the combined samples, two intergenic SNPs, rs1023935 at 4p15.1 and rs79195475 at 10q21.3, were consistently associated with AM. The intron 3 SNP rs3818134 in the SFI1 gene (NM_014775) at 22q12.2 was associated with ANM, with a combined $P$ of $8.8 \times 10^{-6}$ (Table 2).

Replication of previous AM GWAS loci in East Asian women

A total of 110 independent index SNPs at 98 GWAS loci identified from previous studies in women of European ancestry were evaluated in East Asian women; of them, $82(74.5 \%)$ SNPs showed a highly significant concordance of association in the same direction as that found in European-ancestry women $\left(P=2.5 \times 10^{-7}\right.$, binomial sign test; Supplementary Table S3). As shown in Table 3, 22 independent index SNPs representing 19 GWAS loci for AM were nominally replicated in the current study $(P<0.05$ and in the same association direction), a finding highly unlikely to be attributed to chance $(P=2.5 \times$ $10^{-8}$, binomial sign test). The SNP at the Lin-28 Homo$\log$ B gene (LIN28B) locus, rs7759938, had the strongest association $\left(P=3.5 \times 10^{-8}\right.$; Table 3 and Supplementary Table S3). Another independent signal (tagged by rs10453225) within TMEM38B and the SNP rs1400974 downstream of the SATB2 also survived Bonferroni correction for multiple comparisons $\left(P<4.1 \times 10^{-4}\right.$, Table 3).

Replication of previous ANM GWAS loci in East Asian women

Table 4 summarizes the results of 21 independent ANM-associated index/proxy SNPs at 20 GWAS loci previously identified in European-ancestry women.

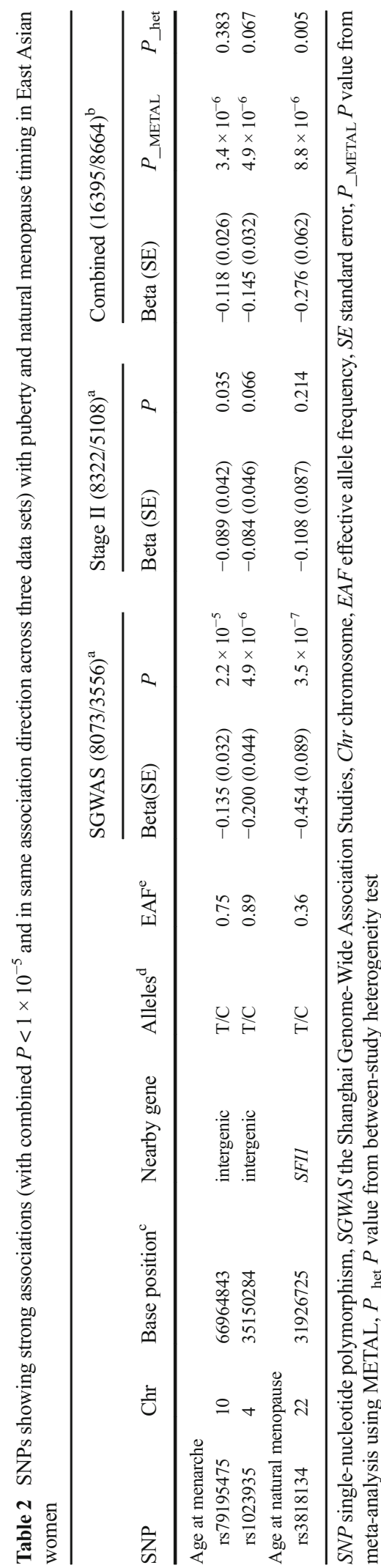




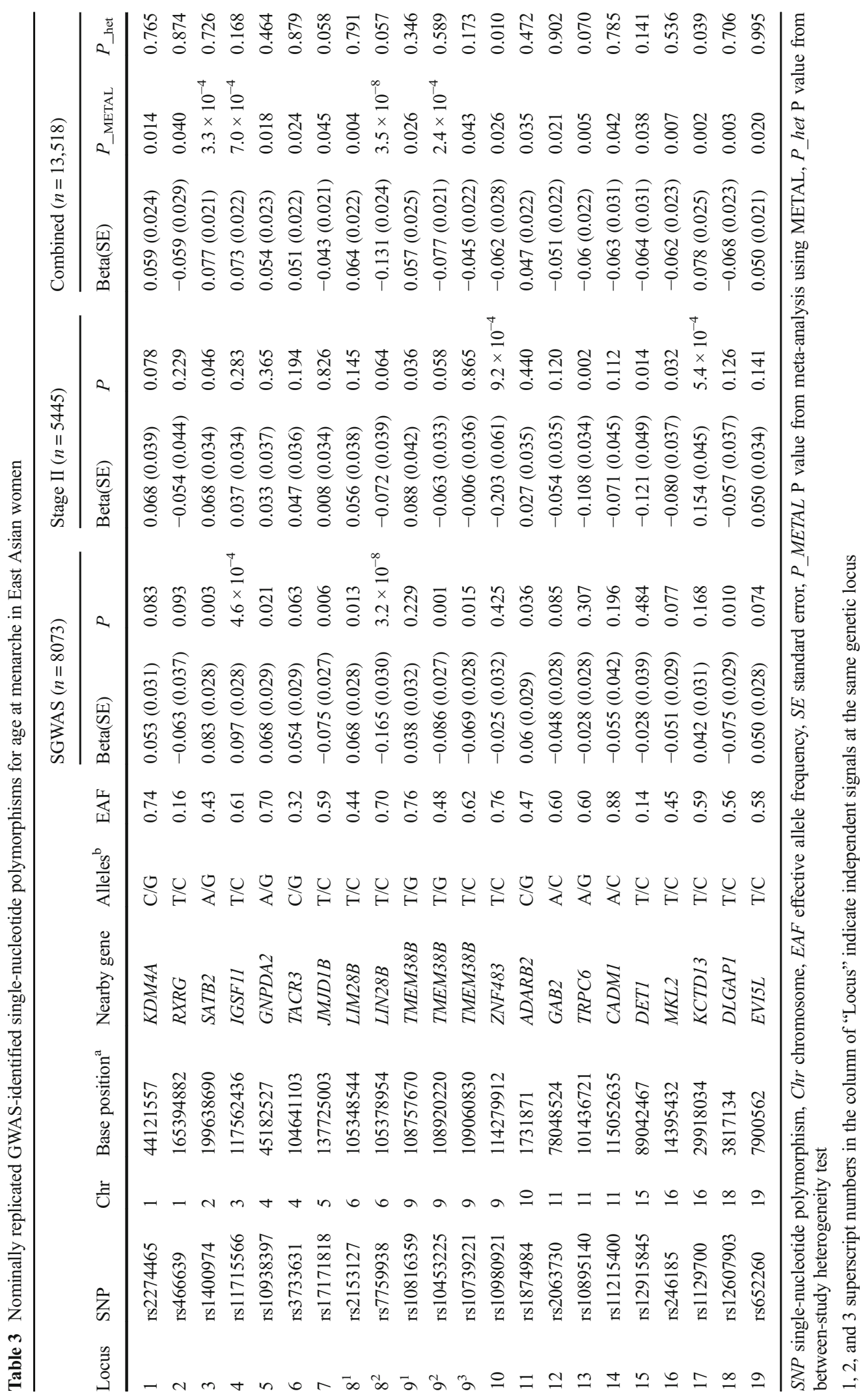




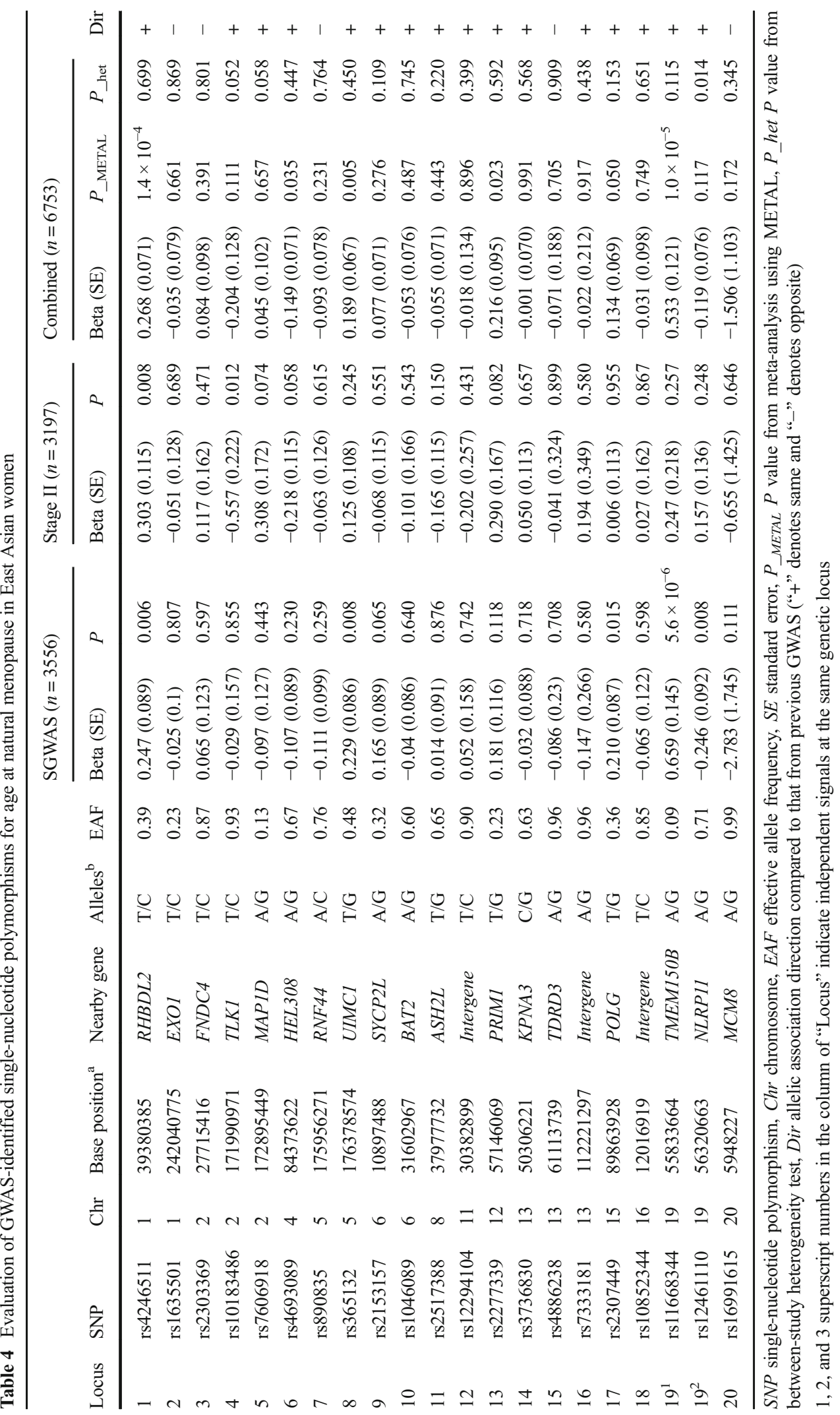


Sixteen SNPs showed a same direction association in East Asian women as that found in European ancestry women $(P=0.027$ for binomial sign test). Five SNPs were nominally replicated (Table 4 ), showing a probability that is significantly higher than what would be expected by chance at the $P<0.05$ level $(P=0.003$, binomial sign test). The association for rs4246511 near the RHBDL2 gene and an intronic SNP rs11668344 in TMEM150B remained significant after Bonferroni correction for 21 independent SNPs evaluated in East Asian women $(P<0.0024)$.

\section{Discussion}

Although limited in statistical power, in this study of up to 16395 East Asian women, we identified two suggestive novel AM loci and replicated 19 of the 98 independent loci for AM, which were previously reported in European ancestry women. In the analysis of up to 8664 East Asian women, we identified one novel suggestive locus and replicated 5 of the 20 independent loci for ANM.

To our knowledge, this study is the first metaanalysis of GWASs in women from two East Asian countries to search for novel genetic loci associated with AM and ANM. Our study also offered a unique opportunity to assess shared genetic determinants of the AM or ANM loci between women of European ancestry and women of East Asian ancestry.

Several limitations in our study deserve mention. First, the relatively small sample size in our study offered a limited statistical power to detect novel genetic loci for these two complex reproductive phenotypes or to replicate most of the previously reported GWAS loci that have a small per-allele effect. For example, under an additive inheritance mode, with allele frequency of 0.25 and perallele effect of 0.123 year-change on AM, our study with 16,395 women has only $46 \%$ power to discover the novel locus 10q21.3 represented by rs79195475 at the genome-wide significance level $\left(P<5.0 \times 10^{-8}\right)$. Power of 13,518 women with available imputed genotype data ranged from 9 to $100 \%$ to replicate GWAS loci with MAF of $0.05-0.50$ and reported per-allele effect between 0.03 and 0.12 years. Second, self-reported AM or ANM may suffer from measurement errors, which further lowered the statistical power of our study. Finally, heterogeneity was observed between studies or sample sets in this two-stage GWAS, although effective allele frequencies are generally comparable across the sample sets
(Tables S1 and S2). We have applied study-specific analyses and adjusted for population stratification in our study to overcome this limitation.

This study highlights a possible novel AM locus near the NKX2-1 gene. Notably, this promising locus at the $N K X 2-1$ gene (Table S1) was also associated with AM in the Japanese population $\left(P=7.4 \times 10^{-6}\right)$ (Tanikawa et al. 2013). Moreover, meta-analysis of SGWAS data and available summary results of four Japanese GWASs confirmed this novel genome-wide significant AM locus in East Asian women (rs2076751: beta of allele $\mathrm{A}=$ -0.105 years, standard error $=0.017, P=1.3 \times 10^{-9}$ ).

Our meta-analysis of summary results from GWAS datasets (not including data of the 2877 samples genotyped using the Sequenom MassARRAY platform) shows a significant association of rs79195475 with AM (beta $=$ -0.145 years, standard error $=0.030, P=1.0 \times 10^{-6}$ ). However, the per-allele effect on AM in the 2877 additional healthy Chinese women from the same population in Shanghai, China, was very low (beta $=-0.012$ years, standard error $=0.054$, not shown in Table 2 ), thus substantially attenuating the association effect from the GWASs and resulting in significant between-study heterogeneity $(P=$ 0.020 ). The suggestive variant $\mathrm{rs} 79195475$ resides in an intergenic region at 10q21.3, which is $707 \mathrm{~kb}$ away from the closest gene CTNNA3 encoding catenin (cadherinassociated protein), alpha 3 . This protein plays a role in cell-cell adhesion in muscle tissue. CTNNA3 gene mutations are thought to cause arrhythmogenic right ventricular cardiomyopathy (van Hengel et al. 2013), and an intron SNP rs12251332 in the CTNNA3 gene has been implicated in heart failure-related serum pyroglutamine level change (Yu et al. 2013). However, whether the suggestive AM locus tagged by rs 79195475 is involved in regulation of CTNNA3, the biological mechanism for its association with AM remains unknown. The second suggestive AM locus, tagged by rs1023935, is mapped to a gene desert region at $4 \mathrm{p} 15.1$. The per-T allele effect in the GWAS ranged from -0.100 in the SeBCS to -0.200 years in the SGWAS; again, its effect size was relatively small (beta = -0.035 ) in the 2877 SWHS women (not shown in Table 2).

For ANM, we identified a suggestive locus at 22q12.2 tagged by rs 3818134 within intron 3 of the SFI1 gene. Notably, each additional copy of the major T allele decreased $0.454 \pm 0.089$ years in menopause age in 3556 women participants in the SGWAS, but only $0.063 \pm$ 0.139 years among 1911 unrelated healthy women from the SWHS (not shown in Table 2). The spindle assemblyassociated Sfil homolog protein encoded by SFI1 
regulates the dynamic structure of centrosome-associated fibers via its interaction with centrin EF-hand protein 2 (Martinez-Sanz et al. 2010). No genetic variants in the SFI1 gene region have been previously reported to be associated with any human disease or trait, including ANM. Therefore, further studies are warranted to investigate this suggestive ANM locus around the SFII gene and functionally support its biological connection to ANM.

By analyzing available data of the East Asian women from China and Korea, we nominally replicated 19 out of the 98 AM loci previously identified through GWAS in European ancestry women (Table 3). The effect sizes of these most-significantly associated loci at LIN28B and TMEM38B were approximately 6.8 and 4.0 weeks, respectively, comparable to those estimated from 182416 European women (Perry et al. 2014). Three independent signals (represented by SNPs rs10816359, rs10453225, and rs10739221) at the TMEM38B gene region and two independent signals (represented by SNPs rs 2153127 and rs7759938) within the LIN28 gene region were nominally replicated $(P<0.05$, Table 3$)$. The third independent signal represented by rs4946632 at the LIN28B locus was not replicated $(P=0.191$, Supplementary Table S3), possibly reflecting allelic heterogeneity among women populations of different ancestry and/or the present under-powered study $(n=13518)$ or a small effect size detected by the European GWAS with a oneorder larger sample size (Perry et al. 2014).

We replicated 5 of the 20 previous GWAS-identified ANM loci at $P<0.05$. These significantly associated SNPs are mapped to the protein-coding genes of RHBDL2, UIMC1, and two closely neighboring genes, BRSK1 and TMEM150B, within the same LD block, respectively. The RHBDL2-encoded human rhomboidlike intra-membrane serine protease could activate epidermal growth factor receptors (Adrain et al. 2011). The coding-synonymous SNP rs365132, in the ubiquitin interaction motif-containing 1 gene (UIMC1), is an expression-quantitative trait locus for nearby genes FGFR4 and ZNF346 in the cortex. UIMC1 functions in DNA repair via interaction with BRCA1 and estrogen receptor $\alpha$ (Stolk et al. 2012). BRSK1 is an AMPactivated protein kinase (AMPK)-related serine/ threonine kinase, which regulates neurotransmitter release at the axonal terminals (Inoue et al. 2006). Transmembrane protein 150B (TMEM150B), also known as TMEM224, belongs to the damage-regulated autophagy modulator family, containing a conserved domain of FGF receptor-activating protein 1 . Although the abovementioned four genes are moderately expressed in human ovaries, no solid evidence indicates a role of their encoded proteins in regulating ovarian aging and thus changing age at menopause.

In conclusion, our genome-wide study of up to 16,395 women of Chinese or Korean ancestry identified two suggestive novel loci for AM and one locus for ANM. In addition, our study nominally replicated 19 AM loci and 5 ANM loci previously identified through GWAS among women of European ancestry. These findings call for larger-scale studies in non-European ancestry populations for identifying additional novel genetic loci and replicating loci for AM and ANM previously identified in women of European ancestry.

Acknowledgments This study was supported, in part, by grants from the US National Institutes of Health (grants R01CA124558, R01CA090899, R01CA070867; R01CA064277 and R01CA092585, R01CA118229, R01CA122756, R01CA137013, R01CA148667), Ingram professorship funds, and Allen Foundation funds at Vanderbilt Epidemiology Center and Division of Epidemiology; SeBCS was supported by the Basic Research Laboratory (BRL) program through the National Research Foundation of Korea funded by the Ministry of Education, Science and Technology (2011-0001564). NHAPC was supported by research grants including the National Basic Research Program of China (2012CB524905 and 2011CB504002), Knowledge Innovation Program (KSCX2-EW-R-10), the National Natural Science Foundation of China (81321062, 81170734, 81471013, 81200581, 81202272), and the Knowledge Innovation Program of Shanghai Institutes for Biological Sciences, Chinese Academy of Sciences (2013KIP107 and 2014KIP107). The authors wish to thank Jing $\mathrm{He}$, Hui Cai, Gong Yang, Jie Wu, and Regina Courtney for their contributions to this project, as well as Nan Kennedy for editing and preparing the manuscript. We also thank the research staff of the Shanghai Breast Cancer Genetics Study, Shanghai Endometrial Cancer Genetics Study, the Shanghai Diabetes Genetics Study, the Shanghai Colorectal Cancer Study, the Nutrition and Health of Aging Population in China study, and the Seoul Breast Cancer Study. In addition, sample preparation and GWAS genotyping assays at Vanderbilt were conducted at the Survey and Biospecimen Shared Resources and the Vanderbilt Microarray Shared Resource, which are supported in part by the VanderbiltIngram Cancer Center (P30CA068485).

\section{Compliance with ethical standards}

Conflict of interest The authors have no conflict of interest to declare. The content is solely the responsibility of the authors and does not necessarily represent the official views of the National Institutes of Health or any other funding agency.

Details of ethics approval Written informed consent was obtained from all participants, and the study protocols were approved by the institutional review boards of all institutions involved in the study. 


\section{References}

Abnet CC, Freedman ND, Hu N, Wang Z, Yu K, Shu X-O, Yuan JM, Zheng W, Dawsey SM, Dong LM et al (2010) A shared susceptibility locus in PLCE1 at 10q23 for gastric adenocarcinoma and esophageal squamous cell carcinoma. Nat Genet 42:764-767. doi:10.1038/ng.649

Adrain C, Strisovsky K, Zettl M, Hu L, Lemberg MK, Freeman M (2011) Mammalian EGF receptor activation by the rhomboid protease RHBDL2. EMBO Rep 12:421-427. doi:10.1038/embor.2011.50

Anderson CA, Zhu G, Falchi M, van den Berg SM, Treloar SA, Spector TD, Martin NG, Boomsma DI, Visscher PM, Montgomery GW (2008) A genome-wide linkage scan for age at menarche in three populations of European descent. J Clin Endocrinol Metab 93:3965-3970. doi:10.1210/jc.2007-2568

Carty CL, Spencer KL, Setiawan VW, Fernandez-Rhodes L, Malinowski J, Buyske S, Young A, Jorgensen NW, Cheng I, Carlson CS et al (2013) Replication of genetic loci for ages at menarche and menopause in the multi-ethnic Population Architecture using Genomics and Epidemiology (PAGE) study. Hum Reprod Oxf Engl 28:1695-1706. doi:10.1093/humrep/det071

Chen CTL, Fernández-Rhodes L, Brzyski RG, Carlson CS, Chen Z, Heiss G, North KE, Woods NF, Rajkovic A, Kooperberg C et al (2012) Replication of loci influencing ages at menarche and menopause in Hispanic women: the Women's Health Initiative SHARe Study. Hum Mol Genet 21:1419-1432. doi:10.1093/hmg/ddr570

Chen CTL, Liu C-T, Chen GK, Andrews JS, Arnold AM, Dreyfus J, Franceschini N, Garcia ME, Kerr KF, Li G et al (2014) Meta-analysis of loci associated with age at natural menopause in African-American women. Hum Mol Genet 23: 3327-3342. doi:10.1093/hmg/ddu041

Cho YS, Go MJ, Kim YJ, Heo JY, Oh JH, Ban H-J, Yoon D, Lee MH, Kim D-J, Park M et al (2009) A large-scale genomewide association study of Asian populations uncovers genetic factors influencing eight quantitative traits. Nat Genet 41: 527-534. doi:10.1038/ng.357

Cramer DW (2012) The epidemiology of endometrial and ovarian cancer. Hematol Oncol Clin North Am 26:1-12. doi:10.1016/j. hoc.2011.10.009

Cui R, Iso H, Toyoshima H, Date C, Yamamoto A, Kikuchi S, Kondo T, Watanabe Y, Koizumi A, Inaba Y et al (2006) Relationships of age at menarche and menopause, and reproductive year with mortality from cardiovascular disease in Japanese postmenopausal women: the JACC study. J Epidemiol Jpn Epidemiol Assoc 16:177-184

De Bruin JP, Bovenhuis H, van Noord PA, Pearson PL, van Arendonk JA, te Velde ER, Kuurman WW, Dorland M (2001) The role of genetic factors in age at natural menopause. Hum Reprod Oxf Engl 16:2014-2018

Delahanty RJ, Beeghly-Fadiel A, Long JR, Gao YT, Lu W, Xiang YB, Zheng Y, Ji BT, Wen WQ, Cai QY et al (2013) Evaluation of GWAS-identified genetic variants for age at menarche among Chinese women. Hum Reprod Oxf Engl 28:1135-1143. doi:10.1093/humrep/det011

Demerath EW, Liu C-T, Franceschini N, Chen G, Palmer JR, Smith EN, Chen CTL, Ambrosone CB, Arnold AM, Bandera EV et al (2013) Genome-wide association study of age at menarche in African-American women. Hum Mol Genet 22:3329-3346. doi:10.1093/hmg/ddt181

Elks CE, Perry JRB, Sulem P, Chasman DI, Franceschini N, He C, Lunetta KL, Visser JA, Byrne EM, Cousminer DL et al (2010) Thirty new loci for age at menarche identified by a meta-analysis of genome-wide association studies. Nat Genet 42:1077-1085. doi:10.1038/ng.714

He C, Kraft P, Chen C, Buring JE, Paré G, Hankinson SE, Chanock SJ, Ridker PM, Hunter DJ, Chasman DI (2009) Genome-wide association studies identify loci associated with age at menarche and age at natural menopause. Nat Genet 41:724-728. doi:10.1038/ng.385

Hinds L, Price J (2010) Menopause, hormone replacement and gynaecological cancers. Menopause Int 16:89-93. doi:10.1258/mi.2010.010018

Howie BN, Donnelly P, Marchini J (2009) A flexible and accurate genotype imputation method for the next generation of genome-wide association studies. PLoS Genet 5:e1000529. doi:10.1371/journal.pgen.1000529

Inoue E, Mochida S, Takagi H, Higa S, Deguchi-Tawarada M, Takao-Rikitsu E, Inoue M, Yao I, Takeuchi K, Kitajima I et al (2006) SAD: a presynaptic kinase associated with synaptic vesicles and the active zone cytomatrix that regulates neurotransmitter release. Neuron 50:261-275. doi:10.1016/j. neuron.2006.03.018

Jia W-H, Zhang B, Matsuo K, Shin A, Xiang Y-B, Jee SH, Kim D-H, Ren Z, Cai Q, Long J et al (2013) Genome-wide association analyses in East Asians identify new susceptibility loci for colorectal cancer. Nat Genet 45:191-196. doi:10.1038/ng.2505

Kim H, Lee J-Y, Sung H, Choi J-Y, Park SK, Lee K-M, Kim YJ, Go MJ, Li L, Cho YS et al (2012) A genome-wide association study identifies a breast cancer risk variant in ERBB4 at 2q34: results from the Seoul Breast Cancer Study. Breast Cancer Res BCR 14:R56. doi:10.1186/bcr3158

Li Y, Willer C, Sanna S, Abecasis G (2009) Genotype imputation. Annu Rev Genomics Hum Genet 10:387-406. doi:10.1146/annurev.genom.9.081307.164242

Li Y, Willer CJ, Ding J, Scheet P, Abecasis GR (2010) MaCH: using sequence and genotype data to estimate haplotypes and unobserved genotypes. Genet Epidemiol 34:816-834. doi:10.1002/gepi.20533

Liu Y-Z, Guo Y-F, Wang L, Tan L-J, Liu X-G, Pei Y-F, Yan H, Xiong D-H, Deng F-Y, Yu N et al (2009) Genome-wide association analyses identify SPOCK as a key novel gene underlying age at menarche. PLoS Genet 5:e1000420. doi:10.1371/journal.pgen.1000420

Long J, Zheng W, Xiang Y-B, Lose F, Thompson D, Tomlinson I, Yu H, Wentzensen N, Lambrechts D, Dörk T et al (2012) Genome-wide association study identifies a possible susceptibility locus for endometrial cancer. Cancer Epidemiol Biomark Prev Publ Am Assoc Cancer Res Cosponsored Am Soc Prev Oncol 21:980-987. doi:10.1158/1055-9965. EPI-11-1160

Marchini J, Howie B, Myers S, McVean G, Donnelly P (2007) A new multipoint method for genome-wide association studies by imputation of genotypes. Nat Genet 39:906-913. doi:10.1038/ng2088

Martinez-Sanz J, Kateb F, Assairi L, Blouquit Y, Bodenhausen G, Abergel D, Mouawad L, Craescu CT (2010) Structure, dynamics and thermodynamics of the human centrin $2 / \mathrm{hSfi} 1$ 
complex. J Mol Biol 395:191-204. doi:10.1016/j. jmb.2009.10.041

Morris DH, Jones ME, Schoemaker MJ, Ashworth A, Swerdlow AJ (2011) Familial concordance for age at natural menopause: results from the Breakthrough Generations Study. Menopause N Y N 18:956-961. doi:10.1097/gme.0b013e31820ed6d2

Murabito JM, Yang Q, Fox C, Wilson PWF, Cupples LA (2005) Heritability of age at natural menopause in the Framingham Heart Study. J Clin Endocrinol Metab 90:3427-3430. doi:10.1210/jc.2005-0181

Ong KK, Elks CE, Li S, Zhao JH, Luan J, Andersen LB, Bingham SA, Brage S, Smith GD, Ekelund U et al (2009) Genetic variation in LIN28B is associated with the timing of puberty. Nat Genet 41:729-733. doi:10.1038/ng.382

Parker SE, Troisi R, Wise LA, Palmer JR, Titus-Ernstoff L, Strohsnitter WC, Hatch EE (2014) Menarche, menopause, years of menstruation, and the incidence of osteoporosis: the influence of prenatal exposure to diethylstilbestrol. J Clin Endocrinol Metab 99:594-601. doi:10.1210/jc.2013-2954

Perry JRB, Stolk L, Franceschini N, Lunetta KL, Zhai G, McArdle PF, Smith AV, Aspelund T, Bandinelli S, Boerwinkle E et al (2009) Meta-analysis of genome-wide association data identifies two loci influencing age at menarche. Nat Genet 41: 648-650. doi:10.1038/ng.386

Perry JRB, Day F, Elks CE, Sulem P, Thompson DJ, Ferreira T, He C, Chasman DI, Esko T, Thorleifsson G et al (2014) Parent-oforigin-specific allelic associations among 106 genomic loci for age at menarche. Nature 514:92-97. doi:10.1038/nature13545

Petersen GM, Amundadottir L, Fuchs CS, Kraft P, StolzenbergSolomon RZ, Jacobs KB, Arslan AA, Bueno-de-Mesquita HB, Gallinger S, Gross M et al (2010) A genome-wide association study identifies pancreatic cancer susceptibility loci on chromosomes 13q22.1, 1q32.1 and 5p15.33. Nat Genet 42:224-228. doi:10.1038/ng.522

Pyun J-A, Kim S, Cho NH, Koh I, Lee J-Y, Shin C, Kwack K (2014) Genome-wide association studies and epistasis analyses of candidate genes related to age at menarche and age at natural menopause in a Korean population. Menopause N Y N 21:522-529. doi:10.1097/GME.0b013e3182a433f7

Qiu C, Chen H, Wen J, Zhu P, Lin F, Huang B, Wu P, Lin Q, Lin Y, Rao H et al (2013) Associations between age at menarche and menopause with cardiovascular disease, diabetes, and osteoporosis in Chinese women. J Clin Endocrinol Metab 98:1612-1621. doi:10.1210/jc.2012-2919

Sharma K (2002) Genetic basis of human female pelvic morphology: a twin study. Am J Phys Anthropol 117:327-333. doi:10.1002/ajpa.10055

Shen C, Delahanty RJ, Gao Y-T, Lu W, Xiang Y-B, Zheng Y, Cai Q, Zheng W, Shu X-O, Long J (2013) Evaluating GWAS-identified SNPs for age at natural menopause among Chinese women. PloS One 8:e58766. doi:10.1371/journal.pone.0058766

Shu XO, Long J, Cai Q, Qi L, Xiang Y-B, Cho YS, Tai ES, Li X, Lin X, Chow W-H et al (2010) Identification of new genetic risk variants for type 2 diabetes. PLoS Genet 6:e1001127. doi:10.1371/journal.pgen.1001127

Spencer KL, Malinowski J, Carty CL, Franceschini N, FernándezRhodes L, Young A, Cheng I, Ritchie MD, Haiman CA, Wilkens L et al (2013) Genetic variation and reproductive timing: African American women from the Population Architecture using Genomics and Epidemiology (PAGE) Study. PloS One 8:e55258. doi:10.1371/journal.pone.0055258
Stolk L, Zhai G, van Meurs JBJ, Verbiest MMPJ, Visser JA, Estrada K, Rivadeneira F, Williams FM, Cherkas L, Deloukas P et al (2009) Loci at chromosomes 13, 19 and 20 influence age at natural menopause. Nat Genet 41:645647. doi:10.1038/ng.387

Stolk L, Perry JRB, Chasman DI, He C, Mangino M, Sulem P, Barbalic M, Broer L, Byrne EM, Ernst F et al (2012) Metaanalyses identify 13 loci associated with age at menopause and highlight DNA repair and immune pathways. Nat Genet 44:260-268. doi:10.1038/ng.1051

Sulem P, Gudbjartsson DF, Rafnar T, Holm H, Olafsdottir EJ, Olafsdottir GH, Jonsson T, Alexandersen P, Feenstra B, Boyd HA et al (2009) Genome-wide association study identifies sequence variants on 6q21 associated with age at menarche. Nat Genet 41:734-738. doi:10.1038/ng.383

Tanikawa C, Okada Y, Takahashi A, Oda K, Kamatani N, Kubo M, Nakamura Y, Matsuda K (2013) Genome wide association study of age at menarche in the Japanese population. PloS One 8:e63821. doi:10.1371/journal.pone.0063821

Van Hengel J, Calore M, Bauce B, Dazzo E, Mazzotti E, De Bortoli M, Lorenzon A, Li Mura IEA, Beffagna G, Rigato I et al (2013) Mutations in the area composita protein $\alpha \mathrm{T}$-catenin are associated with arrhythmogenic right ventricular cardiomyopathy. Eur Heart J 34:201-210. doi:10.1093/eurheartj/ehs373

Velie EM, Nechuta S, Osuch JR (2005) Lifetime reproductive and anthropometric risk factors for breast cancer in postmenopausal women. Breast Dis 24:17-35

Vogel VG (2008) Epidemiology, genetics, and risk evaluation of postmenopausal women at risk of breast cancer. Menopause N Y N 15:782-789. doi:10.1097/gme.0b013e3181788d88

Wen W, Zheng W, Okada Y, Takeuchi F, Tabara Y, Hwang J-Y, Dorajoo R, Li H, Tsai F-J, Yang X et al (2014) Meta-analysis of genome-wide association studies in East Asian-ancestry populations identifies four new loci for body mass index. Hum Mol Genet 23:5492-5504. doi:10.1093/hmg/ddu248

Willer CJ, Li Y, Abecasis GR (2010) METAL: fast and efficient meta-analysis of genomewide association scans. Bioinformatics 26:2190-2191. doi:10.1093/bioinformatics/btq340

Ye X, Yu Z, Li H, Franco OH, Liu Y, Lin X (2007) Distributions of C-reactive protein and its association with metabolic syndrome in middle-aged and older Chinese people. J Am Coll Cardiol 49:1798-1805. doi:10.1016/j.jacc.2007.01.065

Yu B, Zheng Y, Alexander D, Manolio TA, Alonso A, Nettleton JA, Boerwinkle E (2013) Genome-wide association study of a heart failure related metabolomic profile among African Americans in the Atherosclerosis Risk in Communities (ARIC) study. Genet Epidemiol 37:840-845. doi:10.1002/gepi.21752

Zhang B, Jia W-H, Matsuda K, Kweon S-S, Matsuo K, Xiang Y-B, Shin A, Jee SH, Kim D-H, Cai Q et al (2014) Large-scale genetic study in East Asians identifies six new loci associated with colorectal cancer risk. Nat Genet 46:533-542. doi:10.1038/ng.2985

Zheng W, Chow W-H, Yang G, Jin F, Rothman N, Blair A, Li H-L, Wen W, Ji B-T, Li Q et al (2005) The Shanghai Women's Health Study: rationale, study design, and baseline characteristics. Am J Epidemiol 162:1123-1131. doi:10.1093/aje/kwi322

Zheng W, Long J, Gao Y-T, Li C, Zheng Y, Xiang Y-B, Wen W, Levy S, Deming SL, Haines JL et al (2009) Genome-wide association study identifies a new breast cancer susceptibility locus at 6q25.1. Nat Genet 41:324-328. doi:10.1038/ng.318 